\title{
Reflets
}

Revue ontaroise d'intervention sociale et communautaire

\section{Différence chez les personnes âgées : mise en contexte}

\section{Lise Ladouceur}

Volume 2, numéro 2, automne 1996

Vieillir à l'aube de l'an 2000

URI : https://id.erudit.org/iderudit/026131ar

DOI : https://doi.org/10.7202/026131ar

Aller au sommaire du numéro

Éditeur(s)

Reflets : Revue ontaroise d'intervention sociale et communautaire

ISSN

1203-4576 (imprimé)

1712-8498 (numérique)

Découvrir la revue

Citer cet article

Ladouceur, L. (1996). Différence chez les personnes âgées : mise en contexte. Reflets, 2(2), 82-97. https://doi.org/10.7202/026131ar

\section{Résumé de l'article}

La pratique du travail social acquiert sa légitimité en se fondant sur les perceptions, individuelles et collectives, des problèmes sociaux et des personnes qui les vivent à une époque donnée. À l'origine de ces problèmes sociaux, il existe généralement un système organisé de différences selon l'âge, le genre, l'ethnicité, ou encore, les conditions économiques. Nous aborderons les liens entre la vieillesse et les différences à caractères sexuel ou ethnique. Pour ce faire, nous cernerons la situation des femmes âgées francophones vivant en milieu minoritaire. Puis, nous verrons en quoi cette situation interpelle la pratique en travail social.
Tous droits réservés (C) Reflets : Revue ontaroise d'intervention sociale et communautaire, 1996
Ce document est protégé par la loi sur le droit d'auteur. L'utilisation des services d'Érudit (y compris la reproduction) est assujettie à sa politique d'utilisation que vous pouvez consulter en ligne.

https://apropos.erudit.org/fr/usagers/politique-dutilisation/ 


\section{Différence chez les personnes âgées : mise en contexte}

La pratique du travail social acquiert sa légitimité en se fondant sur les perceptions, individuelles et collectives, des problèmes sociaux et des personnes qui les vivent à une époque donnée. À l'origine de ces problèmes sociaux, il existe généralement un système organisé de différences selon l'âge, le genre, l'ethnicité, ou encore, les conditions économiques. Nous aborderons les liens entre la vieillesse et les différences à caractères sexuel ou ethnique. Pource faire, nous cernerons la situation des femmes âgées francophones vivant en milieu minoritaire. Puis, nous verrons en quoi cette situation interpelle la pratique en travail social.

\section{Lise Ladouceur}

Coordonnatrice de projet SHAWN - Social Housing for Abused Women Network à Ottawa et étudiante au doctorat en TS à l'Université Laval de Québec.

\section{Introduction}

Parler de différence, c'est souvent nommer ce qui est distinctif chez une personne. Nos choix de vie, de lieu de résidence ou plus simplement nos goûts alimentaires marquent des différences. Toutefois, les différences sont généralement acceptées par les membres d'une société, car elles ne se présentent pas sous la forme d'un jugement de valeur du genre bon ou mauvais. Le proverbe «des goûts et des couleurs, on ne discute point» l'illustre bien. De même, le fait de vivre en milieu rural plutôt qu'en ville ne remet pas clairement en cause notre identité, ni celle des autres. En fait, ces différences ne font pas l'objet de catégorisation régie par une 
hiérarchie de codes sociétaux bien définis. Ce sont des choix offerts aux membres d'une communauté donnée.

Il importe donc «de faire une distinction entre les différences établies en fonction d'une préférence - la manière dont les gens choisissent de vivre - et les différences constitutives de la personne et fondées sur une inégalité sociale n'offrant guère d'échappatoires» (Juteau-Lee et Roberts, 1981:4, notre traduction). Il sera surtout question ici de ce deuxième type de différences qui construit l'humain en être sexué, ethnicisé et âgé. Avant d'aborder cet aspect dans la pratique, il faut élaborer un cadre théorique. À cette fin, nous puiserons dans le champ conceptuel des catégories de sexe et d'ethnicité, pour ensuite les appliquer aux différences qui caractérisent la vieillesse.

\section{La différence}

"Les humains sont

différents les uns des autres et peuvent ainsi mieux se compléter. Ainsi, les différences enrichissent le monde socialement...»
Le mot différence est polysémique. Pour certains, la différence est utile et nécessaire. Les humains sont différents les uns des autres et peuvent ainsi mieux se compléter. Ainsi, les différences enrichissent le monde socialement, puisque nos différences nous lient plutôt que de nous diviser. En effet, Albert Jacquard, dans son ouvrage L'éloge de la différence, Jacquard (1978), rassemble ses réflexions finales sous le thème «l'amour des différences». Partant d'un bel aphorisme de Saint-Exupéry, «Si je differe de toi, loin de te léser, je t'augmente», (Jacquard, 1978:206) fait une éloquente plaidoirie en faveur de la richesse de la différence (Jouthe, 1993:10).

Dans cet éloge de la différence, il est principalement question d'une différence qui évolue en harmonie. Elle serait moteur de changements sociaux, "source d'information, provocatrice de changements» (Jouthe, 1993:10) Toutefois, une telle vision de la différence n'est pas mise en contexte et n'explique pas les mécanismes qui la font émerger.

La différence n'arrive jamais seule. Pour que la différence existe, 
"Les différences se jouent principalement au niveau des rapports sociaux et ... c'est souvent le regard que l'autre pose sur soi qui vient nous définir.» il faut la comprendre dans un contexte précis. Ce qui nous occupe ici est le contexte social avec tout ce qui le compose. Concrètement, le social représente les interactions des individus entre eux à l'intérieur des organisations sociales. De plus, «le social, c'est le lieu imaginaire de la légitimation réelle des pratiques d'un certain nombre de personnes» (Beynier et al., 1984:13). Le social s'appréhende «lorsque les modalités symboliques de son apparition sont assemblées» (Beynier et al., 1984 :13). Donc, le social n'est jamais un donné figé dans le temps et dans l'espace, il est complexe, toujours à construire ou en construction. Il en est de même pour la différence.

Donner une définition de la différence qui ne tiendrait pas compte du social paraît quelque peu illusoire. Les différences se jouent principalement au niveau des rapports sociaux et, comme Simone De Beauvoir le mentionne, c'est souvent le regard que l'autre pose sur soi qui vient nous définir. Il faut par conséquent inscrire la définition de la différence dans le social.

Dans toute société, nous pouvons remarquer une "division des choses et des activités» (Bourdieu, 1990:8) et des individus. Cette division est arbitraire et repose sur la place qu'occupent ces individus dans la société. Elle présuppose aussi une hiérarchie entre les humains, ce qui rend possible la construction du général et du particulier. Une différence devient alors «une relation dialectique entre deux aspects» (Jouthe, 1993:9) d'une même réalité sociale. Généralement, le groupe des dominants, celui qui possède le pouvoir, est celui qui définira la différence à partir de sa réalité.

Selon Noël (1989), il existe des balises propices à la définition et la diffusion des informations concernant la différence, et elle poursuit en nommant deux lieux d'émergence de la différence : le premier serait propre au «dominant» ou référent, l'autre spécifique aux «dominés» ou différents. Le dominant incarne l'universel qui donne droit de regard sur l'autre. Il utilise la parole universelle inscrite dans le champ de l'histoire, des lois de la nature, dans la volonté de Dieu et dans le savoir construit et diffusé. De plus, Noël présente les mécanismes de légitimation de la différence en termes de dialectique : religion/péché, droit/délit et 
«En ce qui concerne le "dominé», sa personne même - son corps, son identité, sa victimisation - le rend objet de différence.» science/anomalie. En ce qui concerne le «dominé», sa personne même - son corps, son identité, sa victimisation — le rend objet de différence.

C'est essentiellement à partir d'une notion biologique ou somatique et par des habitudes sociales spécifiques, officialisées dans le discours, que la différence prend forme. On passe par l'évident pour construire la différence. On passe principalement par le corps car «le corps est l'indicateur premier du sexe. C'est l'une de ses fonctions sociales que d'actualiser, de rendre visible ce qui est considéré comme la division fondamentale de l'espèce humaine...» (Guillaumin, 1992:117). Lorsqu'on ne peut lire ce corps - comme dans le cas des hermaphrodites - nous avons de la difficulté à attribuer les fonctions sociales «fondamentales» et propres aux sexes biologiques. On se tournera alors vers la médecine pour «convertir» cette anormalité biologique en lieu possible du social, c'est-à-dire que l'on choisit de donner un sexe à cette personne.

Toutefois, le corps, dans ce qu'il représente d'attributs sociaux - porteur d'un sexe, d'une couleur de peau, d'un âge s'engage aussi et surtout dans des rapports sociaux entre hommes et femmes, entre différentes ethnies, entre jeunes et vieux. Il y a,

somatisation progressive des relations fondamentales qui sont constitutives de l'ordre social, [qui] aboutit à l'institution de deux «natures» différentes, c'est-à-dire de deux systèmes de différences sociales naturalisées qui sont inscrits à la fois dans les hexis corporelles, (...) et dans les cerveaux qui les perçoivent selon une série d'oppositions dualistes miraculeusement ajustées à des distinctions qu'elles ont contribué à produire, comme celle que l'on fait entre le droit et la courbe ou le courbé et qui permettrait de réengendrer toutes les différences enregistrées dans l'usage des corps ou dans les dispositions éthiques (Bourdieu, 1990:9,10).

À partir de ce que le corps incarne de «naturel», on construit ce qui doit y être inclus en tant que prescriptions sociales qui, à leur tour, érigeront des comportements propres à chaque groupe. 
Il importe ici de se pencher sur la situation de pouvoir qui fait d'un groupe, le référent, le général, et des autres, le particulier.

Car c'est toujours et seulement le groupe qui regarde de l'extérieur, le nez contre la vitre, dont les caractéristiques et le statut subissent un examen détaillé. C'est ce groupe qui est «différent»,(...) L'absence de pouvoir (...) a le don de faire apparaître ses contestations comme des cas particuliers, et partant faciles à délégitimiser (Patai, 1994:531).

Effectivement, le pouvoir existe sous plusieurs formes. Ici je m'attarde au pouvoir politique (dans le sens large du terme) qui définit la norme. C'est ici qu'entrent en jeu les rapports sociaux qui font du social un lieu biologisé.

Le corps biologique socialement façonné est ainsi un corps politisé, ou, si l'on préfêre, une politique incorporée. Les principes androcentriques sont naturalisés sous la forme de positions et de dispositions élémentaires $d u$ corps qui sont perçues comme des expressions naturelles et de tendances naturelles (Bourdieu, 1990:9,10).

"Ce sont les rapports sociaux qui marquent dans la réalité quotidienne ce que l'imaginaire social a construit à partir du corps.»
Ce sont les rapports sociaux qui marquent dans la réalité quotidienne ce que l'imaginaire social a construit à partir du corps. Mais les rapports sociaux sont souvent évacués lorsqu'il est question de différence. On parle de spécificité des «races», des sexes, d'une particularité naturelle des groupes sociaux. Ainsi, on dit:

qu'une «nature» particulière est directement productrice d'une pratique sociale et [fait] l'impasse sur le rapport social que cette pratique actualise. (...) C'est ainsi que l'esclavage devient un attribut de la couleur de la peau, la non-rémunération du travail domestique un attribut de la forme de sexe (Guillaumin, 1977:49). 
"La différence serait donc un problème pour le groupe qui porte sa marque.»
La différence serait donc un problème pour le groupe qui porte sa marque. Un problème créé par sa "nature» propre telle que définie par le dominant. La différence «n'est [donc] pas un problème pour le groupe non marqué qui est considéré comme la norme» (Patai, 1994 : 530).

Bref, la différence ne peut et ne se limite pas à des traits physiques particuliers ou des habitudes sociales précises. Elle évolue dans le social avec tout ce que cela comporte d'interaction entre les acteurs et le pouvoir qui y est inscrit.

En somme la différence se pense a) dans un rapport, b) mais dans un rapport d'un type particulier où il y a un point fixe, un centre qui ordonne autour de lui et auquel les choses se mesurent, en un mot un référent. (...) La signification idéologique de la différence, c'est la distance au référent. Parler de «différence», c'est énoncer une règle, une loi, une norme. Bref, un absolu qui serait la mesure, l'origine, le point stable du rapport. (...) Il y a un grand réalisme caché dans le mot "différence»: la connaissance qu'il existe une source d'évaluation, un point de repère, une origine de la définition (Guillaumin, $1992: 97)$.

Comme on le voit, penser la différence n'est pas une mince affaire. Pour illustrer ce que je viens de présenter, je vais me servir des différences qui nous touchent de près dans la vie quotidienne, soit les différences basées sur le sexe, l'ethnie pour en arriver à conceptualiser la différence d'âge.

\section{La différence : question de genre ou de race?}

\section{Les sexes}

La dichotomie des sexes semble se fonder sur l'évidence de la «nature». En réalité, on fait «de l'appareil reproducteur externe, 
"...l'homme représente le social, la culture. La femme pour sa part n'est que nature. "

"La femme sera ce qu'elle a en moins et est «une sorte d'entité négative, définie essentiellement par la privation des propriétés masculines et affectée de caractéristiques dépréciatives.» femelle ou mâle, une construction matérielle et symbolique élaborée, destinée à exprimer d'abord, à mettre en valeur ensuite, à séparer enfin les sexes» (Guillaumin, 1992:117). Le danger ici est d'oublier les rapports dans lesquels s'inscrivent les différences sexuelles. "En effet, la vigilance doit être constamment à l'œuvre afin de ne pas ramener implicitement les femmes à leur seule catégorie sexuée, ni de les assigner elles seules comme la seule catégorie sexuée» (Tahon, 1991:2).

Comme le note Françoise Colin, la différence essentielle entre l'homme et la femme a fait l'objet de définition à partir de la spécificité des femmes puisque l'homme

est un particulier qui se vit comme être universel (homo), qui a le monopole, en fait et en droit, de l'humain, c'est-à-dire de l'universel, qui est socialement autorisé à se sentir porteur de la forme entière de l'humaine condition (Bourdieu, 1990:7).

Ainsi l'homme représente le social, la culture. La femme pour sa part n'est que nature. C'est une nature qui représente comment les humains - en l'occurrence les femmes - sont organisés intérieurement pour faire ce qu'ils font et être où ils sont dans la hiérarchie sociale.

Ce naturalisme-là peut s'appeler racisme, il peut s'appeler sexisme, il revient toujours à dire que la Nature, cette nouvelle venue qui prit la place des dieux, fixe les règles sociales et va jusqu'à organiser des programmes génétiques spéciaux pour ceux qui sont socialement dominés (Guillaumin, 1978:5).

Par conséquent, le corps sexué est dépositaire de la catégorie sociale qui sert à la construction du sujet femme, l'homme étant l'humain. La femme sera ce qu'elle a en moins et est «une sorte d'entité négative, définie essentiellement par la privation des propriétés masculines et affectée de caractéristiques dépréciatives» (Bourdieu, 1990:15). 
"Autant d'inégalités basées sur une conceptualisation de la différence qui vise à la supériorité du référent - dans ce cas, les hommes.»
Cette «nature» nous permet d'associer aux évidences somatiques des contraintes naturelles.

Le naturalisme sous-jacent à la conception des femmes s'exprime à un premier degré par l'idée plus ou moins consciente qu'il existe une "naturalité» (...) de la division des tâches entre les sexes, qu'il s'agisse de la «division» du travail ou des tâches dites "reproductives» des femmes (dont les soins aux enfants) - la première étant d'ailleurs sensée découler des secondes (Mathieu, 1991:107).

L'évidence la plus tangible qui découle de la «naturalité» des femmes est la maternité. C'est naturel pour une femme de donner naissance à un enfant, c'est donc «naturel» d'en prendre soin gratuitement.

Des pratiques spécifiques réglementent les rapports sociaux entre les hommes et les femmes et la place de chacun dans la hiérarchie sociale. Pensons seulement aux différences dans les vêtements destinés aux femmes et aux hommes, dans le type de travail réservé aux femmes et les salaires moindres attribués aux femmes. Autant d'inégalités basées sur une conceptualisation de la différence qui vise à la supériorité du référent — dans ce cas, les hommes.

De plus, cette différence «naturelle» des femmes vient jouer au niveau des perceptions que les femmes ont d'elles-mêmes. Elles vivent leur identité de femme à partir du construit social que l'on a fait de leur «nature». Ainsi, l'identité principale d'une femme émerge souvent des rôles qui lui sont dévolus. Elle sera mère et épouse avant tout. Les autres composantes identitaires seront secondaires (c'est du moins ce qu'on tente de nous faire croire encore aujourd'hui).

Il est évident qu'avec l'apparition du féminisme, le rôle des femmes change. Elles sont de plus en plus présentes dans les universités et sur le marché du travail. Toutefois, leur «nature» n'a pas changé pour autant, elle est toujours associée à l'appareil reproducteur qui définit très bien de quoi sont capables les femmes ${ }^{1}$. 


\section{Les races}

Bien que les hommes représentent le général, il existe aussi des «différences». Dans notre contexte nord-américain, les «nègres sont différents (les blancs sont, tout court), les Chinois sont différents (les Européens et les Nord-Américains sont)» (Guillaumin, 1978:16).

"Le racisme relève des mêmes bases théoriques et idéologiques que celles qui produisent le genre.»
Le racisme relève des mêmes bases théoriques et idéologiques que celles qui produisent le genre. On se sert de la «nature» comme fondement de la construction sociale de l'être racial. On proclame donc la matérialité d'un groupe d'humains sur une «nature» fixe et héréditaire.

En gardant une vision «naturaliste» des différences entre les groupes raciaux, on occulte l'importance des relations de pouvoir et des inégalités sociales qui y sont associées. On constate que les membres d'une même société «jouissent de droits et de privilèges inégaux, et qu'une telle inégalité est instituée et rationalisée par une théorie qui allègue la supériorité et l'infériorité naturelle de certaines races» (Bolaria et $\mathrm{Li}, 1988: 14$, notre traduction ${ }^{2}$ ).

La conceptualisation de la différence entre les groupes ethniques sert de mécanisme de hiérarchisation à l'intérieur du social. Effectivement, plus la différence est visible, plus elle marque la place sociale des individus par rapport aux autres.

Le défi premier que doit relever l'individu porteur d'une différence visible est son contact avec les autres. Dès qu'il est vu, en effet, il est non seulement saisi comme "autre», mais comme un être de moindre valeur. Plus la différence est importante, et plus il sera discrédité (...) une marque honteuse, cette différence sera perçue comme révélant une identité elle-même entachée et compromise (Noël, 1989:127).

On peut ainsi constater que la différence est une notion qui affecte nos communautés et les relations sociales entre les groupes humains. Il importe de retenir que la différence n'arrive pas toute seule. Nous sommes toujours différents de quelqu'un, et c'est dans les rapports sociaux qu'évolue la différence. 
"Le sexisme est un essentialisme: comme le racisme, d'ethnie ou de classe...»
«L'âge est aussi une différence fondée sur des phénomènes physiologiques «naturels».»
Ce phénomène de catégorisation et de différenciation donne naissance à des intolérances sociales comme le racisme, le sexisme et l'âgisme.

Le sexisme est un essentialisme: comme le racisme, d'ethnie ou de classe, il vise à imputer des différences sociales historiquement instituées à une nature biologique fonctionnant comme une essence d'où se déduisent implacablement tous les actes de l'existence (Bourdieu, 1990: 12).

\section{La différence basée sur l'âge}

«La vieillesse est une évidence partout présente, partout visible. Témoignages vécus et études savantes en attestent la réalité, la consistance, la matérialité objective et subjective» (Karsz, 1988:33). L'âge est aussi une différence fondée sur des phénomènes physiologiques «naturels». Toutefois, on procède par parcellisation,

qu'il s'agisse de la sociologie de l'enfance (...), de la sociologie de la jeunesse ou de la géronto-sociologie, elles ont choisi un objet dans leur existence sociale, s'attachent aux valeurs et comportements sociaux qui caractérisent cet objet en les expliquant par des facteurs ou des situations sociologiques. Ainsi est-on passé, en ce qui concerne l'âge, de l'idée plus ou moins exprimée qu'il s'agissait là d'un "facteur» individuel psycho-biologique ou psycho-social important à prendre en considération dans les études empiriques, à l'étude proprement sociologique d'une réalité globale propre à chaque «tranche d'âge» (Mathieu, $1991: 19)$.

Bien que l'âge parte d'un donné biologique, sa conceptualisation émerge aussi «du regard extérieur, qu'il vienne de l'entourage et/ou des spécialistes» (Karsz, 1988:36). Ce regard extérieur sanctionne «un ensemble de représentations, définitions et dispositifs à partir duquel le dit sujet est perçu et traité, soit la configuration «vieillesse» dont il est supposé relever» (Karsz, 1988:37). 
"...les pensions de vieillesse et les lois concernant le retrait obligatoire du marché du travail viennent marquer socialement le fait d'être «vieux»ou «vieilles».»

\section{«La situation des} femmes âgées ne déroge pas aux prescriptions sociales qui installent les femmes dans la dépendance vis-à-vis des hommes.»
À la vieillesse, le corps sert de signe visible de la différenciation entre les vieux et les jeunes. C'est une évidence, la sénescence «normale» du corps laisse prévoir l'âge et construit la vieillesse. Par contre, notre représentation corporelle nous introduit et nous maintient dans un espace social qui fait de nous l'autre. Les jeunes, c'est-à-dire les 30-50 ans, eux n'ont pas d'âge .

Le terme «vieillesse» ne se constitue pas exclusivement des transformations physiques, physiologiques et biologiques. Le social et les rapports sociaux viennent se greffer sur cette dimension. En effet, nous sommes confrontés à des politiques sociales qui nous stigmatisent. Ainsi, les pensions de vieillesse et les lois concernant le retrait obligatoire du marché du travail viennent marquer socialement le fait d'être «vieux» ou "vieilles».

«La valeur vieillesse ne s'établit pas en fonction du seul critère de l'âge, 60 ans, 75 ans ou 85 ans, elle ne réside pas dans le nombre, mais dans une opposition: jeune/vieux, actif/passif» (Deremble et Veysset, 1988:57,58). Ainsi, les rapports sociaux tels que nous les connaissons aujourd'hui, où les jeunes «prennent soin» des vieux, ajoutent à notre entendement du terme «vieillesse».

La vieillesse est une catégorie universelle «figure trans-sexuelle, sans clivages, sans classes sociales. (...) Comme l'enfance à la différence des enfants, la vieillesse à la différence des personnes âgées se veut un lieu politiquement et idéologiquement neutre» (Karsz, 1988:44). Par contre, démographiquement, les femmes sont majoritairement représentées dans cette tranche d'âge, plus encore chez les 75 ans et plus.

La situation des femmes âgées ne déroge pas aux prescriptions sociales qui installent les femmes dans la dépendance vis-à-vis des hommes. En effet, la vieillesse ne fait qu'accentuer cette position. En outre, elles ont à faire face à un continuum d'inégalités qui viennent qualifier leur existence à la vieillesse. Par exemple, les femmes qui reçoivent des primes de régime de retraite privé touchent un montant moins élevé que celui des hommes. Effectivement, le salaire des femmes, lorsqu'elles s'insèrent au marché du travail rémunéré, étant moindre que celui des hommes, 
"L'accentuation de la dépendance devient donc un signe important de la vieillesse chez les femmes.» "...en travail

social ... l'image

véhiculée est influencée par les stéréotypes sexistes, racistes et de classe.» les prestations versées au régime de retraite seront moindres, ce qui influence leur revenu. De plus, il faut considérer que les femmes se trouvent souvent dans des secteurs d'activité qui n'offrent pas de pension.

Cette dépendance physique, psychologique et sociale semble vouloir faire des femmes âgées une «race à part», un groupe distinct ayant des traits caractéristiques physiologiques les définissant socialement. "Quand la dépendance apparait, la différence est là, pathologique, déjà intolérable : la différence n'est jamais aussi sensible que lorsque la distance entre deux êtres se réduit au point où l'un pèse sur l'autre sans échappatoire possible» (Deremble et Veysset, 1988:51). L'accentuation de la dépendance devient donc un signe important de la vieillesse chez les femmes.

\section{Le travail social gérontologique}

Le travail social commence tout juste à s'intéresser aux personnes âgées. En fait, le travail social s'attarde beaucoup plus aux conséquences problématiques qui émergent du vieillissement des populations et des individus, puisque l'objet et l'expertise de la profession du travail social sont les problèmes sociaux. Les textes de ce domaine, portant sur cette problématique, présentent une image homogène des personnes âgées.

Toutefois, bien que les écrits en travail social ne distinguent pas les différences individuelles chez les personnes âgées, l'image véhiculée est influencée par les stéréotypes sexistes, racistes et de classe (Hughes et Mtezuka,1992). L'image qui nous est présentée est souvent celle que nous rencontrons quotidiennement dans notre pratique: une personne âgée en perte d'autonomie, «dans une demande de prise en charge diversifiée et en particulier dans le cadre du maintien à domicile» (Pitaud et al., 1988:60).

Il découle de cette vision une difficulté pour le travail social. On ne reconnait pas la place des intervenants sociaux, auprès des «jeunes vieux». Effectivement, «les travailleurs sociaux se sont un peu perdus dans ce qu'ils devaient faire, manquant d'un rôle suffisamment défini, voire même d'une utilité réelle auprès d'une 
"Afin d'intervenir auprès des personnes âgées... on développe une attitude maternante..." "...les femmes âgées ... ont tendance à avoir de la difficulté à obtenir des services sociaux et de soins de santé en français.» population «vieille» de plus en plus jeune par le fait du recours massif à la pré-retraite» (Pitaud et al., 1988:62).

Afin d'intervenir auprès des personnes âgées de façon à trouver une légitimité qui serait propre au travail social, on développe une attitude maternante à l'égard des personnes âgées dépendantes et on en vient «à développer un mécanisme d'ignorance vis-à-vis des jeunes retraités» (Pitaud et al., 1988:65). On effectue ainsi un rapprochement entre la personne âgée et le concept de vieillesse basé sur la notion de «différence».

L'interpellation-vieillesse constitue en fait la mise en auvre discursive et institutionnelle de ce rapprochement entre les personnes âgées et la vieillesse. Sont ainsi proposés des actions concrètes, des améliorations économiques, des initiatives politiques, des accompagnements "sociaux", des suivis thérapeutiques, etc., concernant «le troisième âge» : autant de dispositif susceptibles de raccourcir l'intolérable distance entre ce qui est et ce qui devrait être (Karsz, 1988:44).

La pratique du travail social s'intègre aux structures sociales en place. Les travailleurs sociaux travaillent dans les hôpitaux, les centres de ressources communautaires, centres de jour, etc. Ce sont tous des services d'intégration à une norme associée à la construction sociale de la vieillesse. C'est dans la pratique quotidienne au sein des institutions que les différences basées sur le sexe et l'ethnie viennent jouer un rôle implicite.

Par exemple, les hommes âgés auront accès plus rapidement à des repas à domicile ou aux services domestiques. Ils auront accès aux résidences spécialisées et ils y seront admis à un âge moins avancé que les femmes malgré une meilleure santé physique et mentale (Hughes et Mtezuka, $1992: 234$ ).

En ce qui concerne les femmes âgées francophones en milieu minoritaire, leur situation ressemble à celle de leurs consœurs anglophones. «Toutefois, elles ont tendance à avoir de la difficulté à obtenir des services sociaux et de soins de santé en français. En 
tant que groupe, elles sont également désavantagées sur le plan économique» (Mitchell, 1991 : iii).

\section{Travailler avec les différences}

«Actuellement, la tâche de la majorité des travailleurs sociaux au sein des structures consiste le plus souvent à normaliser, à réconforter, bref, à servir de maillon palliatif aux carences d'une société mal organisée» (Pitaud et al., 1988:72). Mais, l'objet du travail social n'est pas uniquement de rendre service au système. Nous nous devons de trouver des façons, dans notre pratique quotidienne, de travailler auprès d'un groupe en tenant compte des différences des membres qui le composent.

Pour ce faire, Hughes et Mtezuka (1992) proposent de développer une pratique radicale qui prendrait en considération les discriminations sexistes et racistes. Le travail social devrait s'efforcer de reconnaitre l'oppression dûe aux impacts d'une société capitaliste, âgiste et patriarcale sur les femmes âgées (Hughes et Mtezuka,1992). En fait, la pratique du travail social devrait jouer un rôle dénonciateur des discriminations auxquelles les femmes âgées sont confrontées tout en continuant de travailler dans le système (Hughes et Mtezuka, 1992).

En guise de conclusion, soulignons à l'instar de Karsz qu'à la vieillesse,

les différences, clivages et contradictions séparant de fait les personnes âgées ne sont pas toujours effacées ou passées sous silence. Les unes et les autres sont traitées en terme d'écart vis-à-vis du modèle, de réalisations encore défaillantes, de distances restant à colmater. Ils marquent, en bref, ce qu'il en est des personnes âgées en chair et en os: leur incomplétude, leurs failles, à rapprocher autant que possible de leur vieillesse idéale (1988: 43-44). 
La pratique du travail social se trouve à la croisée des chemins entre l'intégration, la normalisation et la dénonciation des valeurs discriminantes qui prévalent dans le système de service social et de la santé. Tenant compte des différences individuelles, il importe de créer des alliances avec les personnes âgées afin de permettre à ces personnes de reprendre le contrôle sur leur vie.

\section{Notes}

1. Encore aujourd'hui, les femmes sont, malgré les brèches dans le filet, majoritairement enfermées dans des ghettos d'emploi. Ces emplois relèvent de leur capacité «naturelle» de prendre soin des autres. Voir Therrien (1987).

2. «Members of society have unequal access to rights and privileges, and that such inequality is institutionalized and rationalized by theory supporting the inherent superiority and inferiority of racial groups (...) (Bolaria et Li, 1988:14).

3. La période de 30 à 50 ans représente le moment intense de participation à l'activité productive, sans qu'on remarque de signes d'une usure extrême. Cette période a été choisie à titre illustratif.

\section{Bibliographie}

BEYNIER, Dominique, Didier LE GALL et Louis MOREAU DE BELLAING, (1984), Analyse du social, Paris, éditions Anthropos.

BOLARIA, Singh, B. PETER et S. LI, (1988), Racial Oppression in Canada, Toronto, Garamond Press.

BOURDIEU, Pierre, (1990), «La domination masculine», dans Actes de recherche en science sociale, no 84, 3-31.

COLIN, Françoise, (1992), «Différence et différent, la question des femmes en philosophie», dans Histoire des femmes, tome V, Le XX $X^{e}$ siècle, sous la direction de Georges Duby et Michelle Perrot, Paris, Éditions Plon, 243-273.

DE BEAUVOIR, Simone, (1970), La vieillesse, Paris, Gallimard.

DEREMBLE Jean-Paul et BernadetteVEYSSET, (1988) «La valeur paradoxale de la vieillesse, dans Les Cahiers de la Recherche sur le Travail Social», no 15, 47-58.

GUILLAUMIN, Colette, (1992), Sexe, race et pratique du pouvoir, L'idée de nature, Paris, éditions Côté-femmes. 
GUILLAUMIN, Colette, (1978), «Pratique du pouvoir et idée de nature. Le discours de la nature», dans Questions féministes, no.3, 5-28.

GUILLAUMIN, Colette, (1977), «Race et nature: système des marques. Idées de groupe naturel et rapports sociaux", dans Pluriel, no 11, 40-56.

HUGHES, Beverley et Melody MTEZUKA, (1992), "Social Work and Older Women: Where Have Older Women Gone?», dans Women and Social Work, edtited by Langan and Day, New York, Routledge.

JOUTHE, Ernst, (1993), «Pour une éthique de la différence en travail social», dans Service Social, vol 42, no 3, 7-19.

JUTEAU-LEE, Danielle, (1979), «La sociologie des frontières ethniques en devenir», dans Frontières ethniques en devenir, Société Canadienne d'études ethniques, Ottawa, Université d'Ottawa, 320.

JUTEAU-LEE, Danielle et Barbara ROBERTS, (1981), «Ethnicity and Feminity (d')après nos expériences", dans Canadian Ethnic Studies/Études ethniques au Canada, vol.XIII, no 1, 1-23.

KARSZ, Saül, (1988), «Déconstruire la vieillesse», dans Les cahiers de la Recherche sur le Travail Social, no $15,33-45$.

MATHIEU, Nicole-Claude, (1991), L'anatomie politique, Paris, éditions Côté-femmes.

MATHIEU, Nicole-Claude, (1990), «Les transgressions du sexe et du genre à la lumière de données ethnographiques», dans Sexe et genre, Marie-Claude Hurtig et al., éditrices, Paris, CNRS, 6980.

MITCHELL, Sheryl, (1991), Les femmes et le vieillissement en Ontario, Toronto, Conseil Consultatif de l'Ontario sur la Condition Féminine.

NELSON, E.D. \& Augie FLERAS, (1995), Social Problems in Canada, Scarborough, Prentice Hall.

NOËL, Lise, (1989), L'intolérance: une problématique générale, Montréal, Boréal.

PATAI, Daphne, (1994), «Le regard d'ailleurs: les constructions utopiques de la «différence»», dans Philosophiques, vol.XXI, no 2, 525-545.

PERROT, Jean, (1983), «La signification économique du travail social», dans Service social dans le monde, déc., 1-13.

PITAUD Ph., A. DEVAUX et R.VERCAUTEREN, (1988), «Les solidarités en gérontologie: une opportunité dans la redéfinition de l'identité professionnelle des travailleurs sociaux?», dans Les cahiers de la Recherche sur le Travail Social, no 15, 59-72.

TAHON, Marie-Blanche, (1991), «Vers une anthropologie politique des femmes», texte de conférence prononcée au Département de sociologie de l'Université d'Ottawa.

THERRIEN, Rita, «Les femmes: productrices et dispensatrices de services sociaux.», dans La contribution informelle des femmes aux services sociaux, présentation dans le cadre de la Commission Rochon, Synthèse critique, no 8.

ZUNIGA, Ricardo et Nicole BOUCHER, (1993), "Autonomie des clients, autonomie des praticiens: les deux visages d'un enjeu social», dans Intervention, no 95, 64-72. 